\title{
Theory of superconductivity in multiwalled carbon nanotubes
}

\author{
E. Perfetto ${ }^{1,2}$ and J. González ${ }^{1}$ \\ ${ }^{1}$ Instituto de Estructura de la Materia, Consejo Superior de Investigaciones Científicas, Serrano 123, 28006 Madrid, Spain \\ ${ }^{2}$ Istituto Nazionale di Fisica Nucleare-Laboratori Nazionali di Frascati, Via E. Fermi 40, 00044 Frascati, Italy
}

(Received 25 September 2006; published 22 November 2006)

\begin{abstract}
We devise an approach to describe the electronic instabilities of doped multiwalled nanotubes, where each shell has in general a manifold of Fermi points. Our analysis relies on the scale dependence of the different scattering processes, showing that a pairing instability arises for a large enough number of Fermi points as a consequence of their particular geometric arrangement. The instability is enhanced by the tunneling of Cooper pairs between nearest shells, giving rise to a transition from the Luttinger liquid to a superconducting state in a wide region of the phase diagram.
\end{abstract}

DOI: 10.1103/PhysRevB.74.201403

PACS number(s): 71.10.Pm, 71.20.Tx, 73.63.Fg, 74.50.+r

Carbon nanotubes (CNs) nowadays offer a great potential for the investigation of mesoscopic physics. Their reduced dimensionality leads to very strong electron correlations, with the consequent breakdown of the conventional Fermi liquid picture. ${ }^{1-4}$ This has become manifest, for instance, in the experimental observation of a power-law suppression of the conductance over a wide range of temperatures. ${ }^{5,6}$ In experiments of a different kind, superconducting (SC) correlations have also been observed in $\mathrm{CNs}$ at low temperatures. There have been measures of a sharp decrease in the resistance of ropes with a large number $(\sim 300)$ of nanotubes, with transition temperatures $T_{c} \sim 1 \mathrm{~K} .{ }^{7,8}$ More recently, quite abrupt drops in the resistance have been measured in large arrays of multiwalled nanotubes (MWNTs) grown in the pores of an alumina template, ${ }^{9}$ with $T_{c} \sim 10 \mathrm{~K}$.

The growth of the SC correlations becomes possible in the CNs when the screened Coulomb interaction is overcome by the effective interaction mediated by phonons. ${ }^{10,11}$ For this to happen, the coupling between a large number of conducting channels is required in general, which favors the screening of the Coulomb interaction. The case of the MWNTs is special in that a certain amount of doping seems to be required to ensure the metallic character of all the shells and the possibility of Cooper-pair tunneling between them. This is consistent with the fact that the drops in the resistance have been only observed in samples where all the shells in the MWNTs are contacted by the electrodes. ${ }^{9}$

The aim of this paper is to study the low-energy instabilities of doped MWNTs, which may have a large number of subbands crossing the Fermi level in each shell. These give rise to a manifold of Fermi points located in two disconnected patches around the $K$ and $K^{\prime}$ points in momentum space, with an approximate circular shape as represented in Fig. 1. For typical shells with radius $R \sim 10 \mathrm{~nm}$, the energy spacing between the subbands is $\Delta E \sim 0.1 \mathrm{eV}$. Below this energy, the different excitations are given by approximate linear branches around each Fermi point, corresponding to the dispersion in the longitudinal momentum $p$. The electronic properties are dictated then by the interaction among a collection of one-dimensional (1D) electron liquids, with electron fields $\psi_{a}(p)$ classified by a Fermi point index $a$. We will take labels that change sign upon inversion in momentum space, with the consequent change of chirality (i.e., right- or left-moving character) of the Fermi point. In this regime, the scattering processes are severely restricted by momentum conservation, and the relevant interactions can be read from the Hamiltonian

$$
\begin{aligned}
H_{\mathrm{int}}= & \int d p d p^{\prime} d q\left[\psi_{a}^{+}(p+q) \psi_{a}(p) f_{a,-b}^{(+)} \psi_{-b}^{+}\left(p^{\prime}-q\right) \psi_{-b}\left(p^{\prime}\right)\right. \\
& +\psi_{-b}^{+}(p+q) \psi_{a}(p) f_{a,-b}^{(-)} \psi_{a}^{+}\left(p^{\prime}-q\right) \psi_{-b}\left(p^{\prime}\right) \\
& +\psi_{b}^{+}(p+q) \psi_{a}(p) c_{a, b}^{(+)} \psi_{-b}^{+}\left(p^{\prime}-q\right) \psi_{-a}\left(p^{\prime}\right) \\
& \left.+\psi_{-b}^{+}(p+q) \psi_{a}(p) c_{a, b}^{(-)} \psi_{b}^{+}\left(p^{\prime}-q\right) \psi_{-a}\left(p^{\prime}\right)\right]
\end{aligned}
$$

where there is an implicit sum over all the pairs of Fermi points $a, b$ with like chirality. ${ }^{12}$ We observe that, as in $2 \mathrm{D}$ systems with a continuous Fermi line, all the relevant interactions can be classified into forward-scattering $\left(f_{a, b}^{(+)}\right)$, exchange $\left(f_{a, b}^{(-)}\right)$, and Cooper-pair $\left(c_{a, b}^{( \pm)}\right)$channels. ${ }^{13,14}$

At the energy scale $\Delta E$, the main contribution to the different couplings in Eq. (1) comes from the Coulomb potential, which in the nanotube geometry is given by

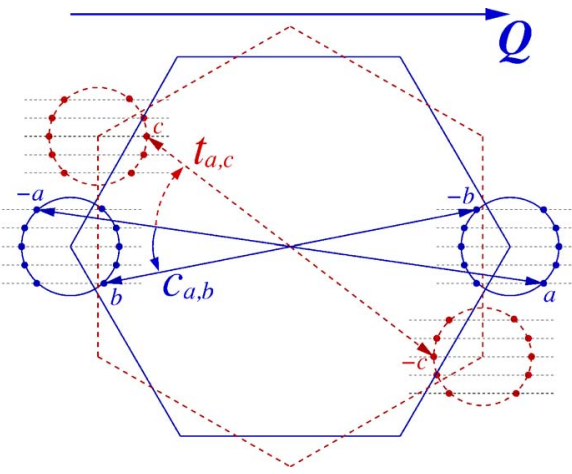

FIG. 1. (Color online) Schematic representation of the location of the Fermi points for the shells of a doped MWNT, referred to the hexagonal Brillouin zone (BZ) of graphene. The horizontal lines correspond to different low-energy subbands, arising from the conical dispersion around the corners of the BZ. The full (dashed) hexagon stands for the case of an armchair (zig-zag) geometry. The $c_{a, b}$ $\left(t_{a, b}\right)$ couplings label the intratube (intertube) scattering of Cooper pairs at zero total momentum. 


$$
V_{C}\left(\mathbf{r}-\mathbf{r}^{\prime}\right)=\frac{e^{2} / \kappa}{\sqrt{\left(x-x^{\prime}\right)^{2}+4 R^{2} \sin ^{2}\left[\left(y-y^{\prime}\right) / 2 R\right]+a_{z}^{2}}},
$$

with $a_{z} \simeq 1.6 \AA$ and $\kappa \approx 2.4 .^{2}$ The Fourier transform of Eq. (2) dictates the value of the couplings $f_{a, b}^{( \pm)}$and $c_{a, b}^{( \pm)}$, according to the corresponding momentum transfer. Thus couplings with momentum transfer of the order of the large momentum Q connecting the $K$ and $K^{\prime}$ points have an intrinsic $1 / R$ dependence, inherent to the behavior of the potential at large momentum. Moreover, in the case of the $f_{a, b}^{(-)}$couplings, the different symmetry of the Bloch wave functions for left- and right-moving electrons leads also to an effective $1 / R$ reduction of their strength. ${ }^{1-3}$ The most important effects of the Coulomb repulsion arise in the forward-scattering interactions, otherwise screened by particle-hole excitations about each of the Fermi points in the different shells. This is consistent with the observation of Luttinger liquid behavior and the relative reduction of the exponent for the power-law dependence of the conductance in doped MWNTs. ${ }^{15,16}$

At energies much lower than $\Delta E$, however, the couplings grow large in general, pointing at the onset of an electronic instability in the system. The character of this instability depends on whether there are different shells electrically active in the MWNTs. Typical MWNTs are made of shells with a random mixture of helicities. In these conditions, the singleelectron tunneling between nearest shells is largely suppressed since, in general, the misalignment of the respective carbon lattices leads to a relative rotation and to the mismatch of the Fermi points, ${ }^{17}$ as shown in Fig. 1. However, such a mismatch does not prevent the intertube tunneling of Cooper pairs with vanishing total momentum. We will take this into account by introducing new intershell tunneling couplings $t_{a, b}^{( \pm)}$, standing for the amplitude of a pair (with electrons around Fermi points $a$ and $-a$ ) to tunnel into a neighbor shell (with electrons around $b$ and $-b$ ). To discern between the possible tunneling of singlet or triplet pairs, we assign an amplitude $t_{a, b}^{(-)}$to the process where the chiralities of the particles in the pair are exchanged, different to that for the direct process $t_{a, b}^{(+)}$.

Below the energy scale $\Delta E$, the electron system is effectively $1 \mathrm{D}$, and the interaction vertices are corrected by diagrams that depend logarithmically on the energy scale $\Lambda$ measured from the Fermi energy $\varepsilon_{F}$. To deal with this divergence, one can integrate progressively the electron modes starting from the limits of the linear branches at $\varepsilon_{F} \pm \Delta E{ }^{18}$ This leads to a dependence of the couplings on the scale $l$ $=-\ln (\Lambda / \Delta E)$, according to the scaling equations

$$
\begin{gathered}
\frac{\partial f_{a, b}^{(+)}}{\partial l}=-\frac{1}{2 \pi v_{a b}}\left[\left(f_{a, b}^{(-)}\right)^{2}-\left(c_{a,-b}^{(+)}\right)^{2}\right], \\
\frac{\partial f_{a, b}^{(-)}}{\partial l}=-\frac{1}{\pi v_{a b}}\left[\left(f_{a, b}^{(-)}\right)^{2}+\left(c_{a,-b}^{(-)}\right)^{2}-c_{a,-b}^{(-)} c_{a,-b}^{(+)}\right],
\end{gathered}
$$

where the $v_{a b}$ are defined in terms of the string of Fermi velocities $v_{a}$ as $v_{a b}=\left(v_{a}+v_{b}\right) / 2$.
The couplings $c_{a, b}^{( \pm)}$are also renormalized by the intertube tunneling amplitudes $t_{a, b}^{( \pm)}$, and their scaling equations turn out to be

$$
\frac{\partial c_{a, b}^{(+)}}{\partial l}=-\sum_{c, s} \frac{1}{2 \pi v_{c}}\left(c_{a, c}^{(s)} c_{c, b}^{(s)}+t_{a, c}^{(s)} t_{c, b}^{(s)}\right)+\frac{1}{2 \pi v_{a b}} c_{a, b}^{(+)} h_{a,-b}^{(+)},
$$

$$
\begin{aligned}
\frac{\partial c_{a, b}^{(-)}}{\partial l}= & -\sum_{c, s} \frac{1}{2 \pi v_{c}}\left(c_{a, c}^{(s)} c_{c, b}^{(-s)}+t_{a, c}^{(s)} t_{c, b}^{(-s)}\right)-\frac{1}{\pi v_{a b}} c_{a, b}^{(-)} h_{a,-b}^{(-)} \\
& -\frac{1}{2 \pi v_{a b}} \sum_{s} c_{a, b}^{(s)} h_{a,-b}^{(-s)}, \\
& \frac{\partial t_{a, b}^{( \pm)}}{\partial l}=-\sum_{c, s} \frac{1}{2 \pi v_{c}}\left(c_{a, c}^{(s)} t_{c, b}^{( \pm s)}+t_{a, c}^{(s)} c_{c, b}^{( \pm s)}\right),
\end{aligned}
$$

with $h_{a,-b}^{(s)} \equiv 2 f_{a,-b}^{(s)}-\delta_{a b} c_{a, a}^{(s)}$.

The above equations apply to the description of MWNTs where all the shells participate in the conduction, as the $t_{a b}^{( \pm)}$ couplings imply the transport between nearest shells. Their relative strength can be estimated by taking the square of the interlayer single-electron hopping in graphite $(\sim 0.1 \mathrm{eV})$, in units of the hopping rate in the graphene lattice $(\sim 2.5 \mathrm{eV})$, leading to relative amplitudes of the order of $\sim 0.002$. When the system has some tendency to develop superconducting correlations, such relatively small couplings at the scale $\Delta E$ are however reinforced at low energies, together with the Cooper-pair couplings. An instability appears then in the electron system at scales which are typically in the range of $l \approx 4-6$. This may be confronted with the instance where only one shell is electrically active, as happens in most experiments with MWNTs. Then the $t_{a, b}^{( \pm)}$couplings have to be taken identical to zero. The flow of Eqs. (3)-(6) still has some unstable regime, but it appears at values of the variable $l \gtrsim 10$, which correspond to temperatures being too low to allow the observation of a new phase (given the appearance of Coulomb blockade effects in real samples at higher temperatures).

We have seen that the contribution of the $e$-e interaction to the intratube couplings at the scale $\Delta E$ is defined exclusively in terms of the potential (2), appropriately screened by the random-phase approximation (RPA) sum of finite particle-hole processes preserving chirality about each of the Fermi points. To complete the evaluation of the couplings, we have added the contribution of the effective phononmediated interaction. We have relied on the results obtained for nanotubes in a wide range of diameters, ${ }^{19,20}$ which coincide in that the coupling $\lambda$ of the effective interaction for small momentum transfer is about three times smaller (in absolute value) than that for momentum about $\mathbf{Q} .{ }^{21}$ We have thus taken a contribution to the original couplings from phonon exchange that scales inversely proportional to the nanotube radius, ${ }^{22}$ with a variation between $\lambda=-0.1 / R$ and $-0.03 / R$ (with $R$ expressed in $\AA$ ) for the channels with small momentum transfer, and respective values equal to $3 \lambda$ for the channels with momentum transfer about $\mathbf{Q} \cdot{ }^{19,20}$ We have ob- 


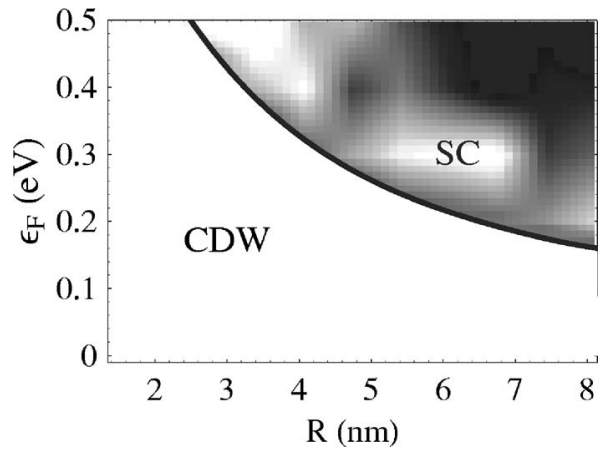

FIG. 2. Phase diagram of doped MWNTs in terms of the average radius $R$ of the shells and the doping level, represented by the shift in the Fermi energy $\varepsilon_{F}$ with respect to the value in the undoped system. The upper region corresponds to a SC phase with $p$-wave symmetry. The density plot represents in gray scale the values of the logarithm of the transition scale $\omega_{c}$, ranging from $-\ln \left(\omega_{c} / \Delta E\right)$ $\approx 4$ (black) to $\approx 6$ (white).

served anyhow that the phase diagram of the system is quite insensitive to the variation of $\lambda$ within that range.

We have characterized the low-energy electronic instabilities by looking at the growth as $\Lambda \rightarrow 0$ of the different response functions, for operators corresponding to order parameters for all types of symmetries [charge-density-wave (CDW), spin-density-wave, superconductivity with various wave symmetries]. The SC response functions are special in that, for a given $g$-wave symmetry, there is the intratube response function $R_{g}(\omega)$ and the intertube counterpart $T_{g}(\omega)$ measuring the propagation of Cooper pairs between nearest shells. The respective derivatives with respect to the frequency, $\bar{R}_{g}=\partial R_{g} / \partial \ln \omega$ and $\bar{T}_{g}=\partial T_{g} / \partial \ln \omega$, satisfy scaling equations ${ }^{18}$

$$
\begin{aligned}
& \frac{\partial \bar{R}_{g}(\Lambda)}{\partial \ln (\Lambda)}=\sum_{a, b, s} g_{a, b}^{(s)} c_{a, b}^{(s)}(\Lambda) \bar{R}_{g}(\Lambda), \\
& \frac{\partial \bar{T}_{g}(\Lambda)}{\partial \ln (\Lambda)}=\sum_{a, b, s} g_{a, b}^{(s)} t_{a, b}^{(s)}(\Lambda) \bar{R}_{g}(\Lambda),
\end{aligned}
$$

where $g_{a, b}^{(s)}$ is the symmetry factor appropriate for each particular wave. We have observed that there is a region of the phase diagram, represented in Fig. 2, where the growth of the $\mathrm{SC}$ response functions is overshadowed by the divergence of any of the CDW response functions at low energies. Above the phase boundary shown in the figure, however, $R_{g}(\omega)$ and $T_{g}(\omega)$ are the only response functions that grow large at low energies. This is the signal that the Cooper pairs are able to propagate across the entire multiwalled structure. The dominant behavior is found in one of the channels of $p$-wave symmetry (see below). We characterize then the transition to the SC state by looking for the frequency scale $\omega_{c}$ marking the onset of the large growth of $T_{g}(\omega)$, before entering its divergent regime.

The boundary between the CDW and the SC phase in the diagram of Fig. 2 corresponds to the situation where the number of Fermi points changes from 12 to 20 in each shell of our model. We have solved the scaling equations for a number of up to $\sim 50$ Fermi points, which already demands the consideration of $\sim 1000$ independent couplings. Then, we have found that the scale $\omega_{c}$ of the SC transition ranges between values corresponding to $l_{c} \equiv-\ln \left(\omega_{c} / \Delta E\right) \approx 4-6$. The accuracy of this prediction relies on the sensible estimate of the couplings at the scale $\Delta E$. In this respect, the SC instability arises essentially from the anisotropic screening of the Coulomb interaction. We have checked for instance that, if $\kappa$ runs between one-half and twice our reference value, there is a variation of $\approx 0.5$ about the original prediction of $l_{c}$. Recalling that $\Delta E \sim 0.1 \mathrm{eV}$, we see that the transition temperatures may reach a higher scale of $\sim 10 \mathrm{~K}$.

There is a natural explanation for the appearance of SC correlations with $p$-wave symmetry, which relies on the peculiar arrangement of the Fermi points. Their discrete distribution reflects the anisotropy in the dimensions of the nanotube, leading to the breakdown of the symmetry of the graphene lattice down to the group $C_{2 v}$. The Cooper-pair vertex, taken as a function of the angular variables $\theta$ and $\theta^{\prime}$ of the pairs before and after the scattering, may be expanded in the form

$$
\begin{aligned}
V\left(\theta, \theta^{\prime}\right)= & V_{0}+V_{1} \cos (\theta) \cos \left(\theta^{\prime}\right)+V_{2} \sin (\theta) \sin \left(\theta^{\prime}\right) \\
& +V_{3} \cos (2 \theta) \cos \left(2 \theta^{\prime}\right)+V_{4} \sin (2 \theta) \sin \left(2 \theta^{\prime}\right)
\end{aligned}
$$

A simple operation allows us to estimate the different coefficients in the expansion. By placing one of the electrons in the incoming pair at the maximum angle $\theta_{0}$ spanned by one circular patch, we may parametrize $V_{1}, V_{2}, V_{3}$, and $V_{4}$ in terms of the values of the vertex at zero momentum transfer, $V\left(\theta_{0}, \theta_{0}\right) \equiv U_{1}$, at $\mathbf{Q}$ momentum transfer, $V\left(\theta_{0}, \pi-\theta_{0}\right) \equiv U_{2}$, with incoming and outgoing electrons at opposite angles, $V\left(\theta_{0},-\theta_{0}\right) \equiv U_{3}$, and at antipodal points, $V\left(\theta_{0}, \pi+\theta_{0}\right) \equiv U_{4}$. We obtain

$$
\begin{gathered}
V_{1} \cos ^{2}\left(\theta_{0}\right)=\left(U_{1}-U_{2}+U_{3}-U_{4}\right) / 4, \\
V_{2} \cos ^{2}\left(\theta_{0}\right)=\left(U_{1}+U_{2}-U_{3}-U_{4}\right) / 4, \\
V_{4} \cos ^{2}\left(2 \theta_{0}\right)=\left(U_{1}-U_{2}-U_{3}+U_{4}\right) / 4 .
\end{gathered}
$$

The key point is that, due to the large number of particle-hole excitations from the many Fermi points, the interactions with very small longitudinal momentum transfer are more screened than those with some transverse (as opposed to longitudinal) momentum within the same circular patch. This means that $U_{1}<U_{3}$, so that an attractive interaction may exist in the Cooper-pair channels with $p$-wave $\left(V_{2}\right)$ and $d$-wave $\left(V_{4}\right)$ symmetry. Moreover, as homologous Fermi points in the two circular patches can be connected by the fixed vector $\mathbf{Q}$, the screening is also enhanced for that particular momentum. This implies that $U_{2}<U_{4}$, leading from Eq. (12) to $V_{2}<0$. This is the origin of the SC instability, as an attractive interaction in one of the pairing channels is always driven to strong coupling in the low-energy limit. ${ }^{13}$

We stress that the disorder introduced by inhomogeneities in the electrostatic potential from the different shells or by 
deformations in a given nanotube cannot have a significant effect on the SC correlations. A remarkable property of the graphene lattice is that defects with a spatial size comparable or larger than the lattice constant give rise to a negligible backscattering in the electron propagation. ${ }^{23}$ Then the effect of such inhomogeneities can be modeled by a random potential coupled to the uniform density fluctuations $\psi_{a}^{+} \psi_{a}$. The SC correlations are actually insensitive to this kind of disorder, since the SC order parameter only depends on the variable (momentum) conjugate to such density fluctuations. ${ }^{24}$ In this perspective, only lattice defects with spatial size smaller than the lattice constant may affect the propagation of the Cooper pairs. Such a kind of disorder may or may not prevent the development of SC correlations depending on whether it reaches some critical strength. ${ }^{24}$ This is consistent with the observations reported in Ref. 9, where the Raman measurements indicate the relative absence of defects in the samples.

We have thus developed an approach that is suitable to describe the electronic instabilities of doped MWNTs, which may have many subbands at the Fermi level. This analysis may be relevant to account for the signatures of SC transitions reported in Ref. 9. Our approach relies on the scale dependence of different scattering processes, and it allows us to describe the transition from the Luttinger liquid to a SC state as the intershell Cooper-pair couplings start to grow large at low energies.
We have seen that the superconductivity in doped MWNTs is based on the anisotropic interactions generated by the particular geometry of the patches of Fermi points. The phonon-mediated interaction plays a minor role in this respect, in agreement with the estimates of its effects when extrapolated to large radius. ${ }^{25}$ The recent observations of critical temperatures of $\approx 11.5 \mathrm{~K}$ in the intercalated compounds of graphite ${ }^{26}$ have also evidenced the weakness of the electron-phonon couplings from carbon bands to account for that effect. ${ }^{27}$ In the MWNTs there is no lattice of intercalants, and the predicted SC instability has a different nature, supported by the quasi-1D character of the electron system. We have seen that the SC state appears in a channel of $p$-wave symmetry, which is otherwise a genuine symmetry of SC instabilities in 1D electron systems, ${ }^{18}$ as observed in the case of the Bechgaard salts. ${ }^{28}$ It would be interesting to check at the experimental level such a quasi-1D character of the superconductivity in the MWNTs, looking for peculiarities already observed in the organic conductors (such as, for instance, the sensitivity of $T_{c}$ to the presence of lattice defects). ${ }^{29}$

The financial support of the Ministerio de Educación y Ciencia (Spain) through Grant No. FIS2005-05478-C02-02 is gratefully acknowledged. E.P. was also supported by INFN Grant No. 10068.
${ }^{1}$ L. Balents and M. P. A. Fisher, Phys. Rev. B 55, R11973 (1997).

${ }^{2}$ R. Egger and A. O. Gogolin, Phys. Rev. Lett. 79, 5082 (1997); Eur. Phys. J. B 3, 281 (1998).

${ }^{3}$ C. Kane, L. Balents, and M. P. A. Fisher, Phys. Rev. Lett. 79, 5086 (1997).

${ }^{4}$ H. Yoshioka and A. A. Odintsov, Phys. Rev. Lett. 82, 374 (1999); A. A. Odintsov and H. Yoshioka, Phys. Rev. B 59, R10457 (1999).

${ }^{5}$ M. Bockrath et al., Nature (London) 397, 598 (1999).

${ }^{6}$ Z. Yao et al., Nature (London) 402, 273 (1999).

${ }^{7}$ A. Yu. Kasumov et al., Science 284, 1508 (1999).

${ }^{8}$ M. Kociak, A. Yu. Kasumov, S. Guéron, B. Reulet, I. I. Khodos, Yu. B. Gorbatov, V. T. Volkov, L. Vaccarini, and H. Bouchiat, Phys. Rev. Lett. 86, 2416 (2001).

${ }^{9}$ I. Takesue, J. Haruyama, N. Kobayashi, S. Chiashi, S. Maruyama, T. Sugai, and H. Shinohara, Phys. Rev. Lett. 96, 057001 (2006).

${ }^{10}$ J. González, Phys. Rev. Lett. 87, 136401 (2001).

${ }^{11}$ J. González, Phys. Rev. Lett. 88, 076403 (2002).

${ }^{12}$ We have not included in Eq. (1) the interactions between uniform density fluctuations $\psi_{a}^{+} \psi_{a}$ with like chirality, as they do not depend on the energy scale and are not relevant therefore for the subsequent scaling analysis.

${ }^{13}$ R. Shankar, Rev. Mod. Phys. 66, 129 (1994); H.-H. Lin, L. Balents, and M. P. A. Fisher, Phys. Rev. B 56, 6569 (1997).

${ }^{14} \mathrm{We}$ will omit spin indices, being implicit that the interactions are independent of the spin orientation. With our notation, forward and exchange scattering can be discerned in practice by their different momentum transfers.
${ }^{15}$ A. Bachtold, M. de Jonge, K. Grove-Rasmussen, P. L. McEuen, M. Buitelaar, and C. Schönenberger, Phys. Rev. Lett. 87, 166801 (2001).

${ }^{16}$ R. Egger, Phys. Rev. Lett. 83, 5547 (1999).

${ }^{17}$ A. A. Maarouf, C. L. Kane, and E. J. Mele, Phys. Rev. B 61, 11156 (2000).

${ }^{18}$ J. Sólyom, Adv. Phys. 28, 201 (1979).

${ }^{19}$ A. Sédéki, L. G. Caron, and C. Bourbonnais, Phys. Rev. B 65, 140515(R) (2002).

${ }^{20}$ D. Connétable, G.-M. Rignanese, J.-C. Charlier, and X. Blase, Phys. Rev. Lett. 94, 015503 (2005).

${ }^{21}$ This relation is also obeyed by the couplings in a graphite layer; see S. Piscanec, M. Lazzeri, F. Mauri, A. C. Ferrari, and J. Robertson, Phys. Rev. Lett. 93, 185503 (2004).

${ }^{22}$ A. De Martino and R. Egger, Phys. Rev. B 67, 235418 (2003).

${ }^{23}$ T. Ando and T. Nakanishi, J. Phys. Soc. Jpn. 67, 1704 (1998).

${ }^{24}$ T. Giamarchi and H. J. Schulz, Phys. Rev. B 37, 325 (1988).

${ }^{25}$ L. X. Benedict, V. H. Crespi, S. G. Louie, and M. L. Cohen, Phys. Rev. B 52, 14935 (1995).

${ }^{26}$ N. Emery, C. Hérold, M. d'Astuto, V. Garcia, Ch. Bellin, J. F. Marêché, P. Lagrange, and G. Loupias, Phys. Rev. Lett. 95, 087003 (2005).

${ }^{27}$ I. I. Mazin, Phys. Rev. Lett. 95, 227001 (2005).

${ }^{28}$ I. J. Lee, S. E. Brown, W. G. Clark, M. J. Strouse, M. J. Naughton, W. Kang, and P. M. Chaikin, Phys. Rev. Lett. 88, 017004 (2001).

${ }^{29}$ A. A. Abrikosov, J. Low Temp. Phys. 53, 359 (1983). 